\title{
The Tile Calorimeter
}

\author{
Olga Bessidskaia* \\ On behalf of the Tile Calorimeter \\ Stockholm University \\ E-mail: obessids@cern.ch
}

The Tile Calorimeter (TileCal) is the the central section of the hadronic calorimeter of the ATLAS experiment. The TileCal is a key in detecting hadrons, jets, hadronic decays of tau leptons and to measure the missing transverse energy. Due to the very good signal to noise ratio it assists the muon spectrometer in the identification and reconstruction of muons. The performance of the calorimeter has been measured and monitored using calibration data, random triggered data, cosmic muons, splash events and LHC collision events. The results presented assess the absolute energy scale calibration precision, the energy and timing uniformity and the synchronization precision. The results demonstrate a very good understanding of the performance of the TileCal that is well within the design expectations.

The XXI International Workshop High Energy Physics and Quantum Field Theory,

June 23 - June 30, 2013

Saint Petersburg Area, Russia

\footnotetext{
*Speaker.
} 


\section{Introduction}

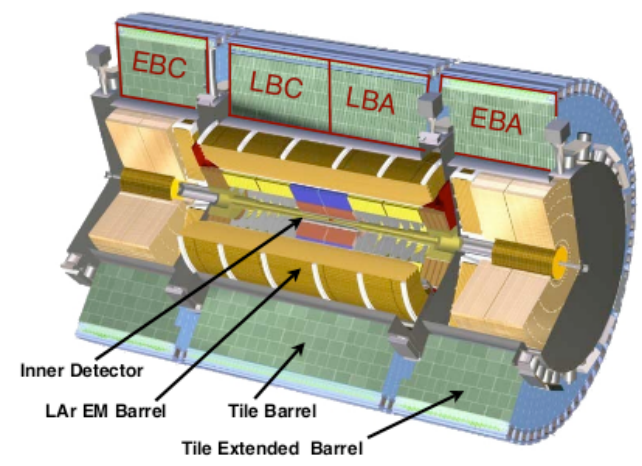

Figure 1: The Tile Calorimeter consists of one long barrel and two extended barrels.

The Tile Calorimeter (TileCal) is the central hadron calorimeter in ATLAS [1], covering ranges in pseudorapidity ${ }^{1}$ of up to $\eta= \pm 1.7$ [2]. TileCal measures the energy and the position of absorbed particles, registering jets, hadronic decays of $\tau$ leptons, and missing transverse energy $E_{T}^{\text {miss }}$. In TileCal, hadrons from collisions can manifest themselves as jets that are built as topoclusters seeded around cells with a large signal compared to noise. The jet energy resolution in TileCal is $50 \% / \sqrt{E[\mathrm{GeV}]} \oplus 3 \%$. The missing transverse energy $E_{T}^{\text {miss }}$ is a quantity that compensates for the apparent non-conservation of transverse momentum in an event. A large $E_{T}^{\text {miss }}$ could indicate to the presence of neutrinos, which do not interact in ATLAS, or it could be a hint of new exotic particles that only interact weakly. TileCal also assists the muon spectrometer in identifying muons.

TileCal is cylindrical in shape, with an inner radius of $228 \mathrm{~cm}$ and an outer radius of $423 \mathrm{~cm}$. It is composed of one long barrel in the middle, of length $654 \mathrm{~cm}$, and two extended barrels on the outside, of length $219 \mathrm{~cm}$ (Fig. 1). A gap of $60 \mathrm{~cm}$ between the barrels allows some space for cables. The extended barrels and the two halves of the long barrel are divided azimuthally (in $\phi$ ) into 64 modules. The mass of TileCal is around 3000 tons.

TileCal is segmented longitudinally into three layers, called A, BC and D cells, as shown in Fig. 2, with 5200 cells in total. The gap between the barrels is partly covered by gap and crack cells, called E cells. The granularity of TileCal is $\Delta \eta \times \Delta \phi=0.1 \times 0.1$ in A and BC cells and $0.2 \times 0.1$ in D cells.

\section{Instrumentation}

Each cell in TileCal consists of dozens of scintillating tiles embedded in steel absorber plates. The plates are positioned perpendicularly to the beam axis. Quarks from collisions in ATLAS hadronize and create showers in the steel in TileCal. The energy deposition is registered as light in the scintillating tiles and transported by wavelength shifting fibres to photomultiplier tubes (PMTs) on the periphery of TileCal, see Fig. 2. Each cell is read out by two PMTs from each $\phi$ side for uniformity and redundancy. The sharp signal is widened by a shaper for better sampling.

\footnotetext{
${ }^{1}$ The definition of the pseudorapidity $\eta$ is $\eta=-\ln \left(\tan \frac{\theta}{2}\right)$, where $\theta$ is the polar angle along the beamline.
} 

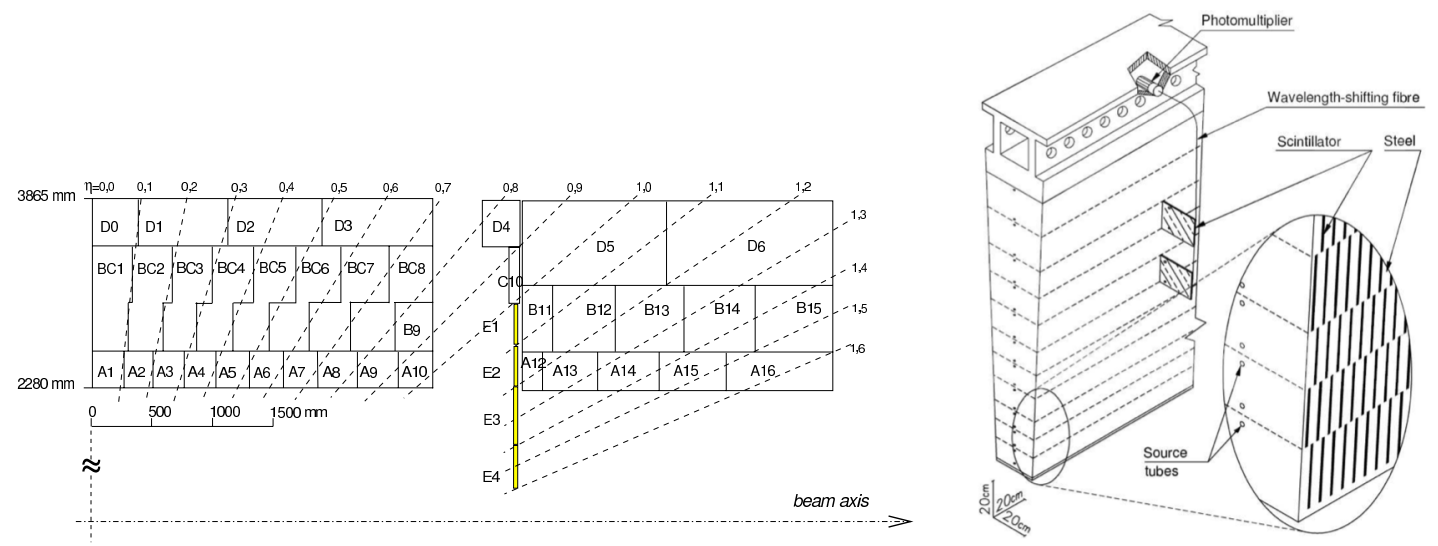

Figure 2: The Tile Calorimeter is segmented longitudinally into A BC and D cells (left). Each barrel is also divided azimuthally into 64 modules. The image to the right shows wavelength shifting fibres transporting light from the tiles in a module to the PMTs.
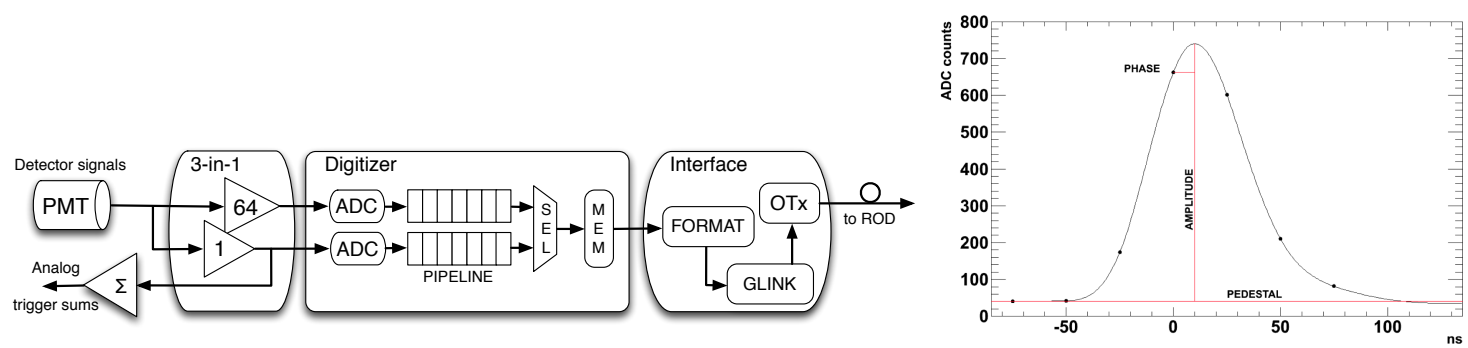

Figure 3: The front end electronics in TileCal (left). After shaping and digitization, each signal consists of seven samples, $25 \mathrm{~ns}$ apart (right).

The pulse is amplified in high gain and low gain branches with a gain ratio of 64 in a 3-in-1 card. The 3-in-1 card provides a signal to the adder board, which in turn provides an analog trigger signal. In parallel with communication with the trigger, the signal is digitized by a 10-bit ADC into 7 samples 25 ns apart, as shown in Fig. 3 (right). From the amplitude of such a pulse, the deposited energy can be derived. The position of the peak gives the timing. The digital signals are stored in front end pipeline memories, where they await the level 1 trigger decision. Signals that are given a level 1 trigger accept are transported from the pipeline memory to the Read Out Drivers (RODs) for reconstruction. The schematics of the front end electronics are shown in Fig. 3 (left).

\section{Calibration}

The signal from the ADCs needs to be related to the charge deposited in the PMTs and translated into the energy deposited in the cell. This is accomplished by using conversion factors from a Charge Injection System (CIS) to convert between charge and ADC counts, and with conversion factors from testbeams to relate the charge to an energy. During CIS calibration of the front-end electronics, a charge is injected into the 3-in-1 card and the signal is read out by the RODs. The RMS of the variation in response to charge injection is $0.08 \%$ over the year 2012 [3]. As the CIS 
constants are very stable, they are only updated twice a year. Nevertheless, charge injection runs are performed weekly for monitoring and identifying so-called bad channels.

In addition, the change over time in the response of the system to energy deposition needs to be accounted for. To do so, calibrations with laser light and with a Cesium source are performed. The Cesium system is used for monthly calibrations of the system. Sources of radioactive ${ }^{137} \mathrm{Cs}$ transverse all the tiles through metallic pipes. A hydraulic system transports the sources through the barrels. The integrated current read out from the PMTs as the Cesium passes by is used to correct for variations in the conversion factors mentioned above. The calibration precision with Cesium is $0.4 \%$ [4]. The response of the PMTs to light is monitored by illumination of the PMTs with laser. This provides constants that adjust for changes in between the Cesium calibrations. During data taking, weekly laser calibration runs are performed. The precision of the laser calibration is $0.3 \%$ [5]. The average down-drift of the PMT response due to ageing is at most $1.3 \%$ a year, E cells excluded, as seen in Fig. 4 (right). Periods of data taking are visible in Fig. 4 (left) as a down drift of the laser response. During periods of mainentance, when there are no collisions, the response of the PMTs recovers.
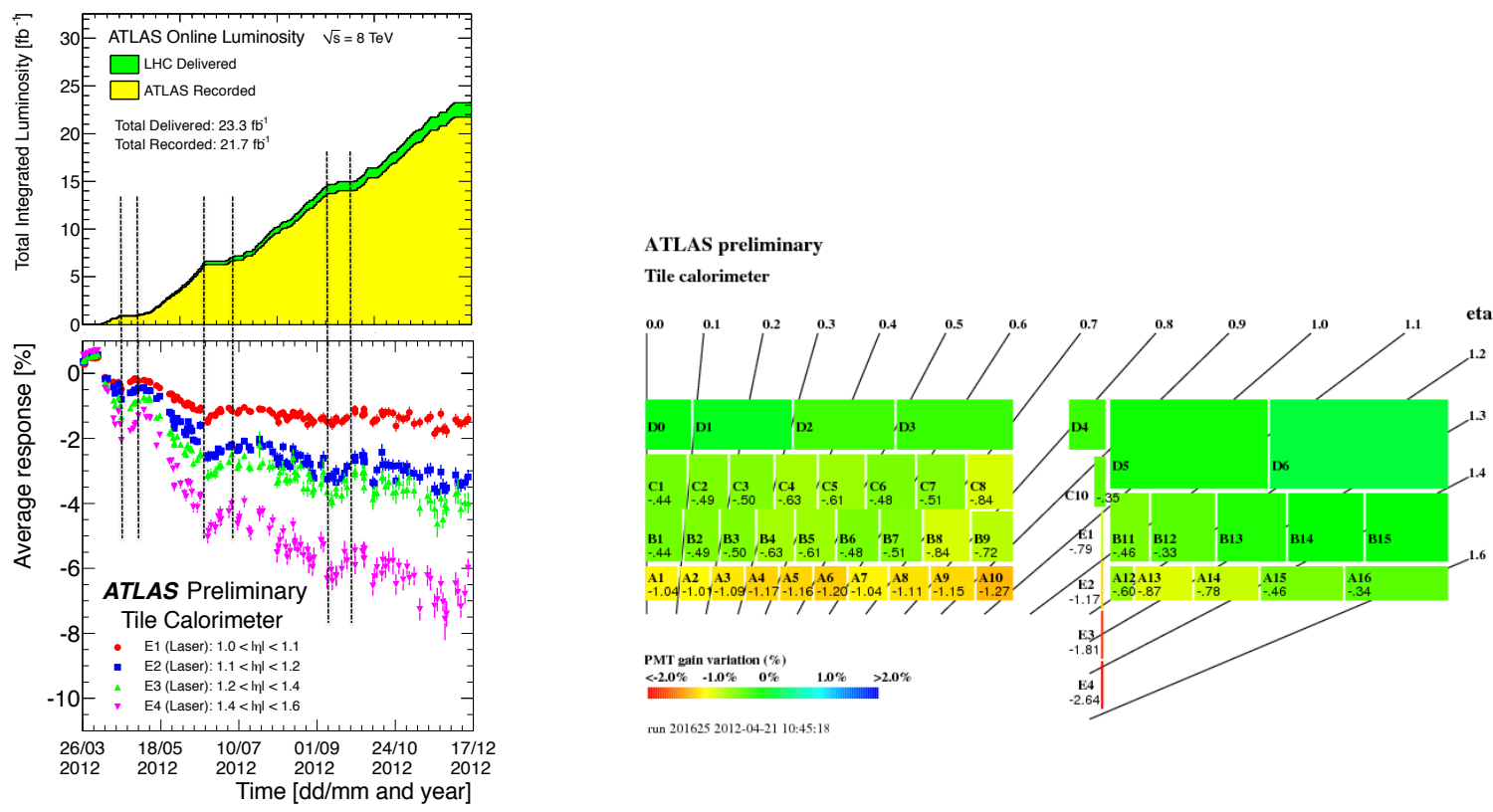

Figure 4: The response of the cells to laser light from the calibration system is shown to the left. The response of the $\mathrm{E}$ cells reflects periods of data taking and maintenance. The the variation in response to laser light in per cent over one year is seen to the right.

\section{Performance}

Good precision in timing is crucial for correct jet energy reconstruction as the time between two consecutive bunch crossings is $50 \mathrm{~ns}$. For a bunch spacing of $25 \mathrm{~ns}$, as is planned for Run 2 , good timing will be indispensable. The precision in timing derived from collision data is 0.85 ns [6]. The cell time resolution is below $1 \mathrm{~ns}$ when the jet energy is greater than $4 \mathrm{GeV}$, as shown in Fig. 5. 


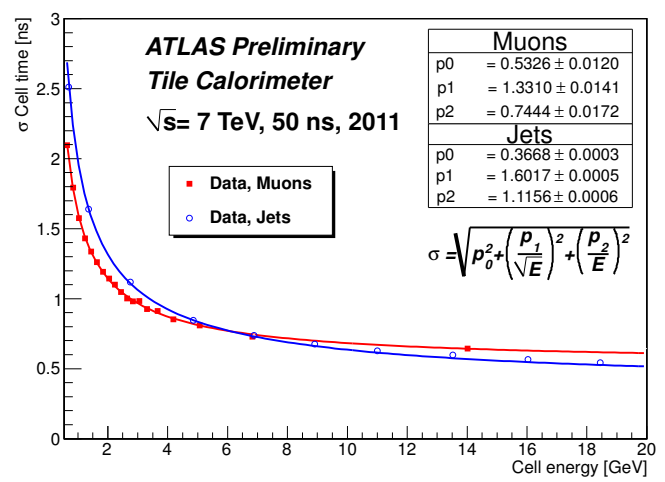

Figure 5: The timing resolution as a function of energy.
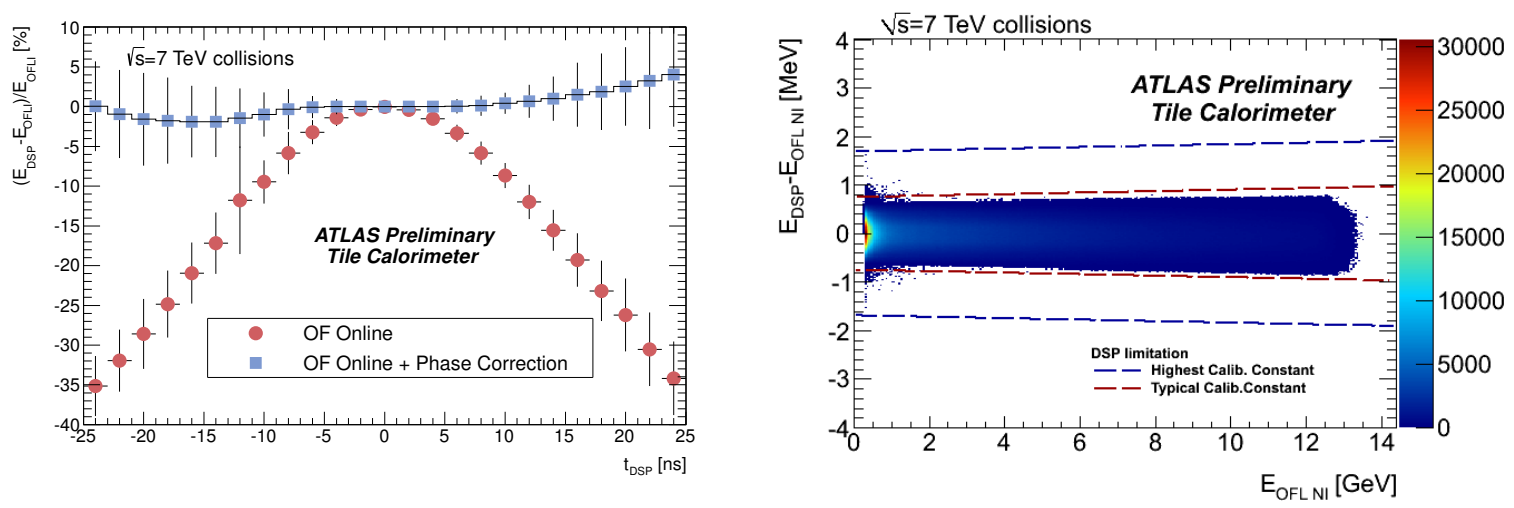

Figure 6: The energy reconstruction with two different methods (left). After correction for timing (blue), the two methods agree well. The difference in reconstructed energy (right) by two different methods as a function of the energy from offline reconstruction.

A validation of the energy reconstruction is performed by comparison of two different methods, the noninterative method and the offline iterative method, as shown in Fig. 6 (left). Corrections for timing are applied offline for better energy reconstruction, which is demonstrated by the consistency between the two methods shown by the blue points. In total, $99 \%$ of channels fall within the maximum expected precision in energy reconstruction of $\pm 1 \mathrm{MeV}$, as can be seen in Fig. 6 (right). The maximum expected difference between the two is proportional to the calibration constants.

The data quality efficiency is high for TileCal, with a data loss of below $0.1 \%$ of the integrated luminosity in 2012. The largest sources of data taking losses were the problems with the readout system and with timing, as seen from Fig. 7 (left). These issues were solved successfully. Overall, TileCal is $99.6 \%$ efficient, as presented in Fig. 8.

Cells in TileCal that deliver corrupted data are masked. The number of masked cells throughout 2012 is shown in Fig. 7 (right), with periods of maintenance shown in red. Maintenance efforts brought the fraction of masked cells to per mille level each time. The number of masked cells was $2.93 \%$ at end of data taking. A sharp increase of masked cells corresponds to a loss of an entire 

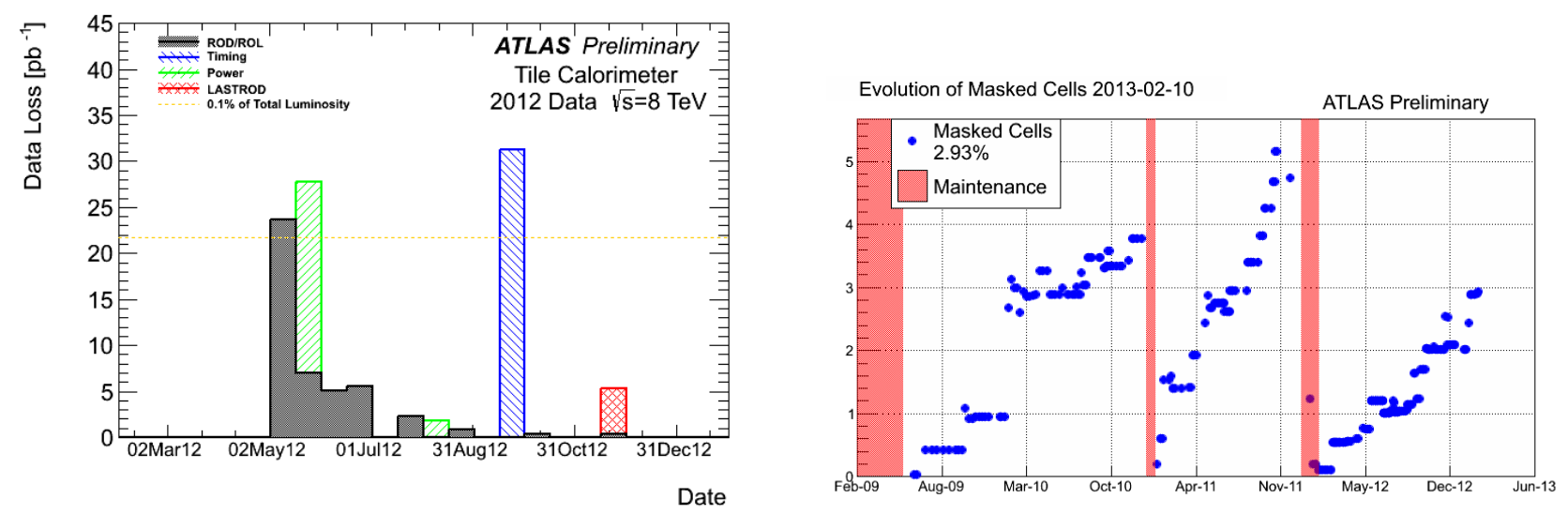

Figure 7: The loss of data in $\mathrm{pb}^{-1}$ over the year of 2012 (left). The orange line indicates a loss of $0.1 \%$ of the luminosity. The percentage of masked cells in TileCal through 2012 is shown to the right.

\begin{tabular}{|c|c|c|c|c|c|c|c|c|c|c|}
\hline \multicolumn{11}{|c|}{ ATLAS p-p run: April-December 2012} \\
\hline \multicolumn{3}{|c|}{ Inner Tracker } & \multicolumn{2}{|c|}{ Calorimeters } & \multicolumn{4}{|c|}{ Muon Spectrometer } & \multicolumn{2}{|c|}{ Magnets } \\
\hline Pixel & SCT & TRT & LAr & Tile & MDT & RPC & $\operatorname{CSC}$ & TGC & Solenoid & Toroid \\
\hline 99.9 & 99.1 & 99.8 & 99.1 & 99.6 & 99.6 & 99.8 & 100. & 99.6 & 99.8 & 99.5 \\
\hline \multicolumn{11}{|c|}{ All good for physics: $95.5 \%$} \\
\hline
\end{tabular}

Figure 8: The efficiency of the different detectors in ATLAS are shown here. TileCal was $99.6 \%$ efficient for the period April-December 2012.

module or a half of a module, for example due to the breakdown of Low Voltage Power Supplies (LVPS) or power connectors. The LVPS can also experience trips. The number of trips in the LVPS is proportional to the integrated luminosity, as demonstrated in Fig. 9 (left), with a total of 14000 trips in 2012.

Yet another problem with the LVPS in 2012 was related to the noise. The electronic noise in TileCal is caused by random fluctuaions in the electronics. The modelling of the noise has so far been a double Guassian, as the long tails in the distribution make a single-Gaussian description inaccurate. Replacing the LVPS with a newer version results in a narrower distribution (i.e. lower noise) that also looks more single-Gaussian, as shown in Fig. 9 (right).

\section{Outlook}

At the present we are in a period called long shutdown, lasting from February 2013 to April 2015. During this time, there are no collisions and the detectors can be repaired and improved. In TileCal, the drawers containing the electronics are being consolidated and the LVPS are replaced to reduce power trips and noise.

An upgrade [7] of the hardware is planned in 10 years time. All signals are to be digitized at $40 \mathrm{MHz}$ in the front-end electronics and all such digital samples will be transmitted to the trigger 

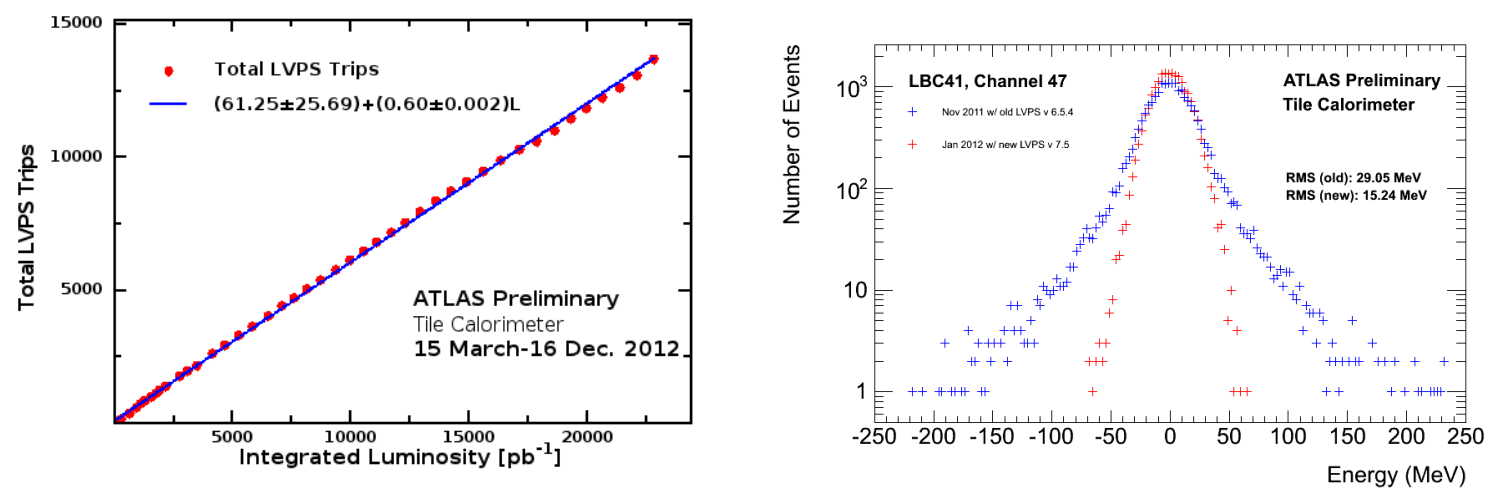

Figure 9: The number of LVPS trips as a function of the luminosity (left). The noise is described by a double Gaussian (right), to account for the long tails seen in the blue distribution. In red we see the noise for the same channel with a new LVPS. The noise is smaller and the long tails disappear.

in back-end electronics. This is in contrast with the present situation, when an analog signal is provided to the trigger while digital samples are stored in pipeline memories in the front-end. The pipeline memories are to be moved to the back-end electronics to protect them from the higher radiation levels expected from the higher rate of bunch crossings in the next run period.

To summarize, the calibration systems in TileCal have a remarkable precision and follow the design requirements. More than $99.9 \%$ of delivered luminosity was recorded in 2012. Before the long shutdown, $97.1 \%$ of the cells in TileCal were operational. At present, a massive consolidation work is performed to reduce variations and prevent data loss and an upgrade of the hardware is planned. Overall, the Tile Calorimeter performs well, delivering reliable data to the physics analysis.

\section{References}

[1] The ATLAS Collaboration et al, The ATLAS Experiment at the CERN Large Hadron Collider, JINST 3 (2008) S08003.

[2] The ATLAS Collaboration, Readiness of the Tile Calorimeter for LHC collsions, July 2010, Eur.Phys.J.C70:1193-1236,2010.

[3] Wesserman, N. D and Dulchinos, N, Atlas TileCal Performance Meeting (31/01/2013), twiki.cern.ch/twiki/bin/view/AtlasPublic/ ApprovedPlotsTileCalibration\#Charge_injection_system_CIS, Feb 2013.

[4] The ATLAS Collaboration, Readiness of the ATLAS Tile Calorimeter for LHC collisions, Nov 2010, Eur.Phys.J.C70:1193-1236,2010, arXiv:1007.5423.

[5] Boumediene, D and Dybreuil,E, twiki.cern.ch/twiki/bin/view/AtlasPublic/ ApprovedPlotsTileCalibration\#Laser, Dec 2012.

[6] Fiorini, L and Davidek, T and Succurro, A, ATLAS Collaboration, TileCal public plots 2010 Collisions, Mar 2011.

[7] Cerqueira, A. S, ATLAS Tile Calorimeter Readout Electronics Upgrade Program for the High Luminosity LHC, May 2013, arXiv:1305.0859. 